\title{
Functional Link Artificial Neural Network for Denoising of Image
}

\author{
Charu Pandey ,Vartika Singh ,O.P.Singh ,Satish Kumar \\ Deptt. of Electronics \& Electrical Engineering (ASET), Amity University, Uttar Pradesh, Lucknow
}

\begin{abstract}
Digital image denoising is crucial part of image preprocessing. The application of denoising procesn satellite image data and also in television broadcasting. Image data sets collected by image sensors are generally contaminated by noise. Furthermore, noise can be introduced by transmission errors and compression. Thus, denoising is often a necessary and the first step to be taken before the images data is analyzed. In this paper a Modified Functional Link Artificial Neural Network (M-FLANN) is proposed which is simpler than a Multilayer Perceptron (MLP). It have been implemented for image restoration in this paper. Its computational complexity and speed and generalization ability to cancel Gaussian noise is compared with that of MLP. In the single layer functional link ANN (FLANN) the need of hidden layer is eliminated. The novelty of the FLANN structure is that it requires much less computation than that of MLP. In the presence of additive white Gaussian noise, salt and pepper noise, Random variable impulse noise and mixed noise in the image the performance of the proposed network is compared with that of MLP in this paper. The Performance of the of algorithm is evaluated for six different situations i.e. for single layer neural network, MLP and four different types of expansion in FLANN and comparison in terms of computational complexity also carried out.
\end{abstract}

Index Terms-MLP, FLANN, Salt and Pepper noise.

\section{Introduction}

The quality of digital medical images has become an important issue today. To achieve the best possible diagnoses, it is important for medical images to be sharp, clear, and free of noise and artifacts [1]. Though, the technologies used to improve resolution and quality of noisy images remains an issue in many medical images applications. Removing noise in these digital images remains as one of the major challenges in the study of medical imaging. Denoising of ultrasound images is particularly challenging due to their peculiar texture [3]. The presence of noise will degrade the quality of image, and even conceal image details, which affects the subsequent image segmentation, feature extraction and recognition, quantitative analysis, and most importantly disease diagnosis. A computationally efficient artificial neural network (ANN) for the purpose of dynamic nonlinear system identification is proposed[6]. The major drawback of feed forward neural networks, such as multilayer perceptrons (MLPs) trained with the back propagation (BP) algorithm, is that they require a large amount of computation for learning. We propose a single-layer functional-link ANN (FLANN) in which the need for a hidden layer is eliminated by expanding the input pattern by Chebyshev polynomials. The novelty of this network is that it requires much less computation than that of a MLP. We have shown its effectiveness in the problem of nonlinear dynamic system identification. In the presence of additive Gaussian noise, the performance of the proposed network is found to be similar or superior to that of a MLP. A performance comparison in terms of computational complexity has also been carried out.

Recently artificial neural network (ANN) has emerged as a powerful learning technique to perform complex tasks in highly nonlinear environment [9]. The advantages of ANN model are due to there ability to learn based on optimization of an appropriate error function and there excellent performance for approximation of nonlinear functions.

The functional link artificial neural network (FLANN) by Pao can be used for function approximation and pattern classification with faster convergence and lesser computational complexity than a MLP network. A FLANN using sin and cos function for functional expansion for the problem of nonlinear dynamic system identification has been reported [13].A single layer orthogonal neural network using Legendre polynomials has been reported for static function approximation. In this work we proposed a FLANN structure similar to a Chebyshev polynomial-based unified model ANN for de-noising in an image corrupted with different noises[7]. Generally a linear node in its output is used in the FLANN structure reported by other researchers.

This paper tries to improve the time complexity involved in training the network, along with minimizing the number of interconnection weights and biases that are used as the network parameters which make it suitable for on-line application in comparison to MLP. This technique is able to approximate linear as well as non linear functions. This is computationally cheap and has small convergence time[3]. It de-noises image more efficiently. 


\section{Real-life applications of ANN}

The tasks artificial neural networks are applied to tend to fall within the following broad categories:

a) Function approximation, or regression analysis, including time series prediction, fitness approximation and modeling.

b)Classification, including pattern and sequence recognition, novelty detection and sequential decision making.

c) Data processing, including filtering, clustering, blind source separation and compression.

d) Robotics, including directing manipulators, Computer numerical control.

\section{Structure Of The Artificial Neural Network}

Here, we briefly describe the architecture and learning algorithm for multilayer neural network and FLANN.

\section{A. Multilayer perceptron}

The MLP has a multilayer architecture with one or more hidden layers between its input and output layers. All the nodes of a lower layer are connected with all the nodes of the adjacent layer through a set of weights. All the nodes in all layers (except the input layer) of the MLP contain a nonlinear $\tanh ($ ) function. A pattern is applied to the input layer, but no computation takes place in this layer[4]. Thus the output of the nodes of this layer is the input pattern itself. The weighted sum of outputs of a lower layer is passed through the nonlinear function of a node in the upper layer to produce its output. Thus, the outputs of all the nodes of the network are computed. The outputs of the output layer are compared with a target pattern associated with the input pattern[5]. The error between the target pattern and the output layer node is used to update the weights of the network. The MSE is used as a cost function and BP algorithm attempts to minimize the cost function by updating all weights of the network[6].

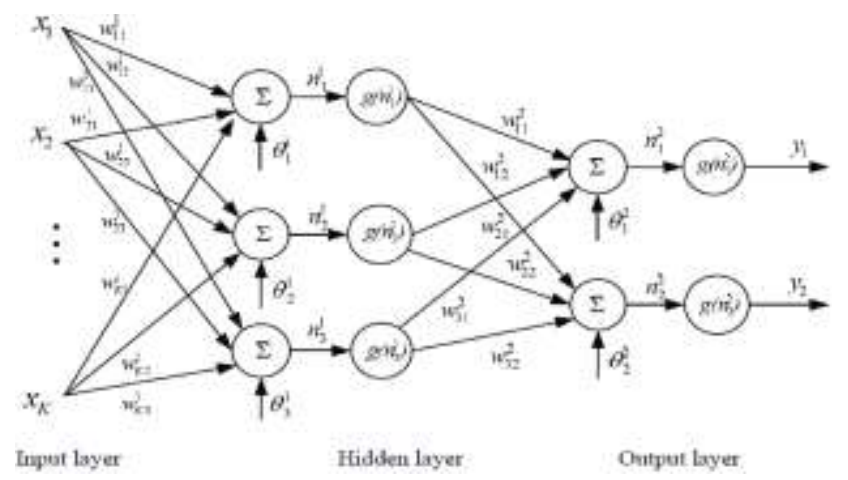

Figure 1: Multilayer perceptron neural network architecture

\section{B. Functional link ANN}

The FLANN, which is initially proposed by Pao, is a

single layer artificial neural network structure capable of performing complex decision regions by generating nonlinear decision boundaries[9]. In a FLANN the need of hidden layer is removed. In contrast to linear weighting of the input pattern produced by the linear links of a MLP, the functional link acts on the entire pattern by generating a set of linearly independent functions.

Here the functional expansion block comprises of exponential polynomials. Suppose input to this structure is $\mathrm{X}=[\mathrm{x} 1 \mathrm{x} 2]^{\prime}$. An enhanced pattern obtained by using functional expansion given by $\mathrm{X}=[1 \mathrm{x} 1 \mathrm{~T} 2(\mathrm{x}) \ldots \ldots \mathrm{x} 2 \mathrm{~T} 2(\mathrm{x} 2) \ldots \ldots .$.

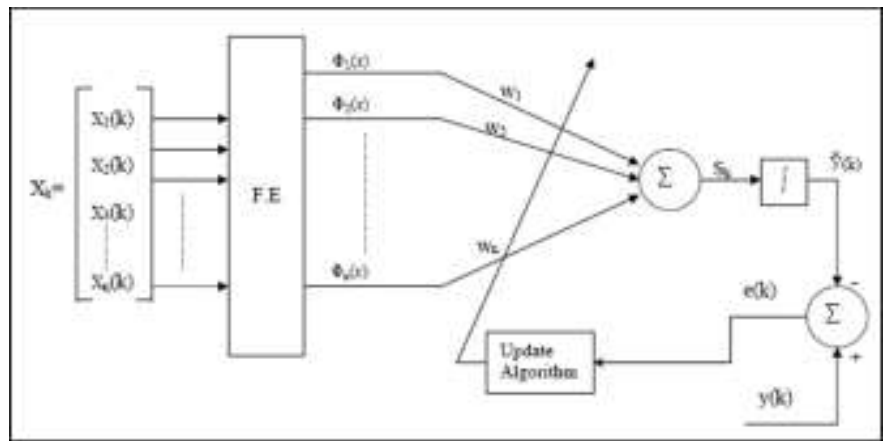

F.E: - Functional Expansion

Figure 2: Structure of FLANN with a single output 


\section{a) Learning with the FLANN}

Let there be K number of input-output pattern pairs to be learned by the FLANN. Let the input pattern vector $\mathrm{X}$ is of dimension and for ease of understanding, let the output, $\mathrm{y}$ be a scalar. Each of the input patterns is passed through a functional expansion block producing a corresponding $\mathrm{N}$-dimensional $(\mathrm{N}>=\mathrm{n})$ expanded vector.

In this case, the dimension of the weight matrix is of $1 \mathrm{x} \mathrm{N}$ and hence, the individual weights are represented by a single subscript[10]. Let $\mathrm{w}=[\mathrm{w} 1 \mathrm{w} 2 \ldots . \mathrm{wN}]$ be the weight vector of its FLANN. The linear weighted sum, Sk is passed through the tanh (.) nonlinear function to produce the output $\breve{y}(\mathrm{k})$ with the following relationship:

$$
\hat{y}_{k}=\tanh \left(S_{k}\right) \text { or } S_{k}=\frac{1}{2} \log _{\theta}\left(\frac{1+\hat{y}_{k}}{1-\hat{y}_{k}}\right)
$$

\section{b) The Learning Algorithm}

Let $\mathrm{K}$ number of patterns be applied to the network in a sequence repeatedly. Let the training sequence be denoted by $\{\mathrm{Xk}, \mathrm{Yk}\}$ and the weight of the network be $\mathrm{W}(\mathrm{k})$, where $\mathrm{k}$ is the discrete time index given by $\mathrm{k}=\mathrm{k}+\lambda \mathrm{k}$, for $\lambda=0,1,2 \ldots$, and $\mathrm{k}=0,1,2 \ldots, \mathrm{K}$. At $\mathrm{kth}$ instant, the $\mathrm{n}$-dimensional input pattern and the $\mathrm{m}$-dimensional FLANN output are given by

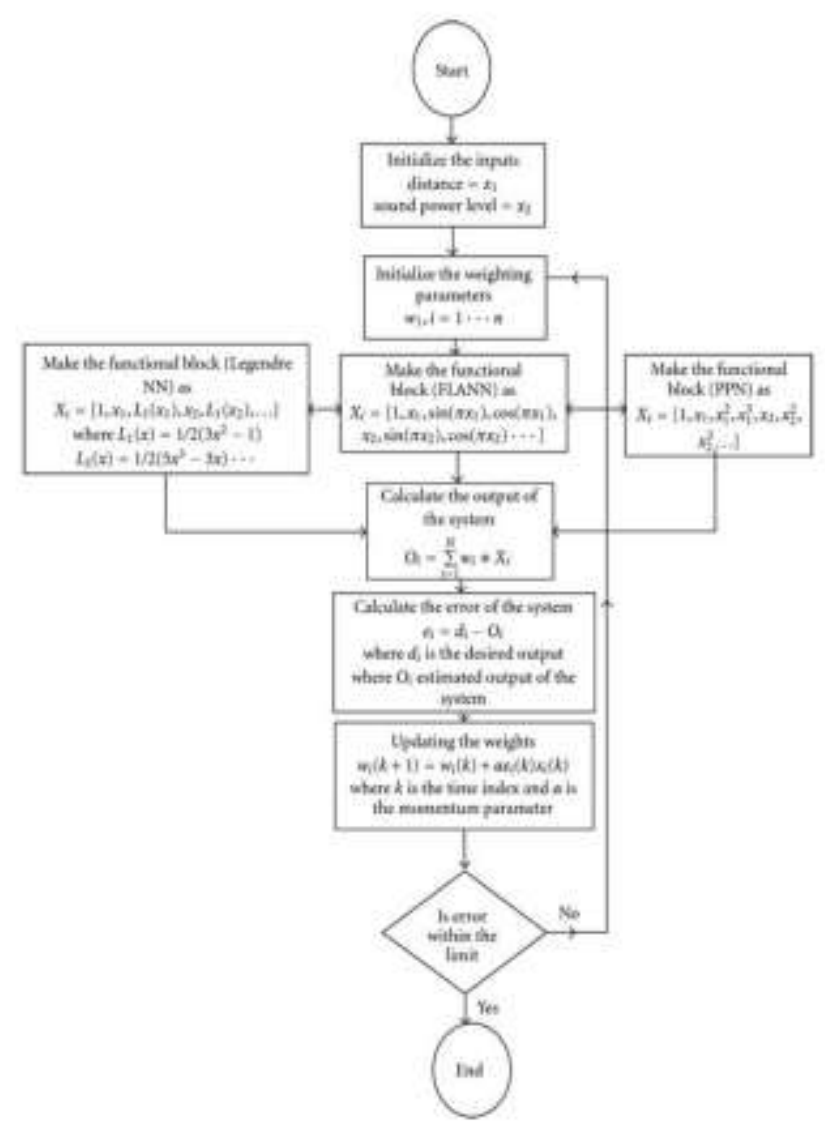

Figure 3: Systematic algorithm of functional based neural network

$$
\begin{gathered}
\mathrm{Xk}=[\mathrm{x} 1(\mathrm{k}) \mathrm{x} 2(\mathrm{k}) \ldots \ldots \ldots \mathrm{xn}(\mathrm{k})] \mathrm{T} \text { and } \\
\breve{\mathrm{y}}(\mathrm{k})=[\mathrm{Y} 1(\mathrm{k}) \mathrm{Y̆} 2(\mathrm{k}) \ldots \ldots . . . \mathrm{Y̆} \mathrm{m}(\mathrm{k})] \mathrm{T} \text { respectively. }
\end{gathered}
$$

Its corresponding target pattern is represented by

$$
\mathrm{Y}(\mathrm{k})=[\mathrm{y} 1(\mathrm{k}) \mathrm{y} 2(\mathrm{k}) \ldots \ldots \ldots \mathrm{ym}(\mathrm{k})] \mathrm{T} .
$$

The dimension of input pattern increases from $\mathrm{n}$ to $\mathrm{N}$ by a basis function $\Phi$ given by 


$$
\Phi(\mathrm{Xk})=[\Phi 1(\mathrm{Xk}) \Phi 2(\mathrm{Xk}) \ldots \ldots . . \Phi \mathrm{N}(\mathrm{Xk})] \mathrm{T}
$$

The $(\mathrm{mxN})$ dimensional weight matrix is given by

$$
\mathrm{W}(\mathrm{k})=[\mathrm{W} 1(\mathrm{k}) \mathrm{W} 2(\mathrm{k}) \ldots \ldots . \mathrm{Wm}(\mathrm{k})] \mathrm{T}
$$

Where $\mathrm{Wj}(\mathrm{k})$ is the weight vector associated with jth output and is given by

$$
\mathrm{Wj}(\mathrm{k})=[\mathrm{Wj} 1(\mathrm{k}) \mathrm{Wj} 2(\mathrm{k}) \ldots \ldots . . . \mathrm{WjN}(\mathrm{k})]
$$

The jth output of the FLANN is given by

$$
\hat{y}_{j}(k)=\rho\left(\sum_{i=1}^{N} w_{j t}(k) \phi\left(X_{k}\right)\right)
$$

for $\mathrm{j}=1,2,3 \ldots \mathrm{m}$

\section{c) Trigonometric Functional Expansion}

Here the functional expansion block make use of a functional model comprising of a subset of orthogonal sin and cos basis functions and the original pattern along with its outer products. For example, consider a two dimensional input pattern $\mathrm{X}=[\mathrm{x} 1 \times 2] \mathrm{T}$, the enhanced pattern is obtained by using a trigonometric functions as $\mathrm{x}^{\prime}=[\mathrm{x} 1 \cos (\pi \times 1) \sin (\pi \times 1) \cos (3 \pi \times 1) \sin (3 \pi \times 1)]$. The LMS algorithm, which is used to train the network, becomes very simple because of the absence of any hidden layer.

\section{d) Chebyshev Expansion}

The Chebyshev polynomials are a set of orthogonal polynomials defined as the solution to the Chebyshev differential equation and denoted as $\operatorname{Tn}(\mathrm{x})$. These higher the first few Chebyshev polynomials are given by

$$
T_{0}(x)=1.0, T_{1}(x)=x \text { and } T_{2}(x)=2 x^{\wedge} 2-1
$$

The higher order Chebyshev polynomials may be generated by the recursive formula given by:

$$
T_{n+1}(x)=2 x T_{n}(x)-T_{n-1}(x)
$$

The first few Chebyshev polynomials are given by

$$
\begin{aligned}
& \mathrm{T} 0(\mathrm{x})=1 \\
& \mathrm{~T} 1(\mathrm{x})=\mathrm{x} \\
& \mathrm{T} 2(\mathrm{x})=2 \mathrm{x} 2-1 \\
& \mathrm{~T} 3(\mathrm{x})=4 \mathrm{x} 3-3 \mathrm{x} \\
& \mathrm{T} 4(\mathrm{x})=8 \mathrm{x} 4-8 \mathrm{x} 2+1 \\
& \mathrm{~T} 5(\mathrm{x})=16 \mathrm{x} 5-20 \mathrm{x} 3+5 \mathrm{x}
\end{aligned}
$$

\subsubsection{Exponential expansion}

In this study we used exponential polynomials for functional expansion as shown in figure-1. These polynomials are easy to compute than that of other polynomials. For two-dimensional input patterns, the enhanced pattern is obtained by using exponential functions as:

$\mathrm{X} 1=[\mathrm{x} 1 \exp 1 \exp 2 \mathrm{x} 1 \exp 3 \mathrm{x} 1 \ldots \mathrm{x} 2 \exp \mathrm{2} 2 \exp 2 \times 2 \exp 3 \times 2 \exp 4 \times 2 \ldots.] \mathrm{T}$.

This type of polynomial expansion needs less number of computations and is very easy to implement than other three types of polynomial expansion. 


\section{Computational Complexity}

Here, we present a comparison of the computational complexity between an MLP, and a FLANN having different expansions and all having the $\tanh ($.$) as their nonlinear function. In all the cases, multiplications, additions$ and computations of the $\tanh ($.$) are required. However in the case of FLANN, additional computations of the sine and$ cosine functions are needed for its functional expansion. In the training and updating of the weights of MLP, extra computations are incurred due to its hidden layer. This is due to the error propagation for the calculation of the square error derivative of each neuron in the hidden

layer. For each iteration the computation are: (1) Forward calculations to find the activation value of all the nodes of the entire network.(2) Back-error propagation for calculation of square error derivatives.(3) Updating weights of the entire network. In the case of MLP with $\{\mathrm{I}-\mathrm{J}-\mathrm{K}\}$, the total number of weights is given by $(\mathrm{I}+1) \mathrm{J}+$ $(\mathrm{J}+1) \mathrm{K}$. Whereas, in the case of FLANN with $\{\mathrm{D}-\mathrm{K}\}$, it is given by $(\mathrm{D}+1) \mathrm{K}$. The number of computation for both MLP and FLANN are shown in table:

TABLE

COMPARISON OF COMPUTATIONAL COMPLEXITY IN ONE ITERATION

\begin{tabular}{|c|l|l|}
\hline Operation & \multicolumn{1}{|c|}{$\begin{array}{c}\text { MLP } \\
\{\mathrm{I}-\mathrm{J}-\mathrm{K}\}\end{array}$} & \multicolumn{1}{|c|}{$\begin{array}{c}\text { FLANN } \\
\{\mathrm{D}-\mathrm{K}\}\end{array}$} \\
\hline Addition & $2 \mathrm{~J}+3 \mathrm{JK}+$ & $2 \mathrm{~K}(\mathrm{D}+1)+$ \\
& $3 \mathrm{~K}$ & $\mathrm{~K}$ \\
\hline Multiplication & $3 \mathrm{~J}+4 \mathrm{JK}+$ & $3 \mathrm{~K}(\mathrm{D}+1)+$ \\
& $3 \mathrm{~J}+5 \mathrm{~K}$ & $2 \mathrm{~K}$ \\
\hline Tanh(.) & $\mathrm{J}+\mathrm{K}$ & $\mathrm{K}$ \\
\hline
\end{tabular}

From this table it may be seen that the number of additions, multiplications and computation of tanh are much less in case of a FLANN than that of a MLP network. As the number of hidden layer increases the computations in a MLP increases. But due to absence of hidden layer in the FLANN its computational complexity reduces drastically.

\section{Experiment-1}

\section{Simulation Results}

TABLE:

\begin{tabular}{|c|c|c|c|c|c|}
\hline $\begin{array}{l}\text { Gaussi } \\
\text { an } \\
\text { Noise } \\
\text { Mean } \\
\text { zero } \\
\text { and } \\
\text { Varian } \\
\text { ce }\end{array}$ & $\begin{array}{l}\text { Noise } \\
\text { Powe } \\
\mathrm{r} \text { at } \\
\text { Input } \\
\text { of } \\
\text { FLA } \\
\text { NN } \\
\text { Filter }\end{array}$ & $\begin{array}{l}\text { Noise } \\
\text { powe } \\
\mathrm{r} \text { at } \\
\text { outpu } \\
\mathrm{t} \text { of } \\
\text { FlAN } \\
\mathrm{N} \\
\text { Filter }\end{array}$ & $\begin{array}{l}\text { PSNR } \\
\text { at } \\
\text { Input } \\
\text { of } \\
\text { FLA } \\
\text { NN } \\
\text { Filter }\end{array}$ & $\begin{array}{l}\text { PSNR } \\
\text { at } \\
\text { Outpu } \\
\text { t of } \\
\text { FLA } \\
\text { NN } \\
\text { Filter }\end{array}$ & $\begin{array}{l}\text { Noise } \\
\text { Power } \\
\text { Attenua } \\
\text { ted } \\
\text { By the } \\
\text { Filter in } \\
\text { dB }\end{array}$ \\
\hline 0.01 & $\begin{array}{l}0.042 \\
9\end{array}$ & $\begin{array}{l}0.007 \\
62\end{array}$ & $\begin{array}{l}16.54 \\
56\end{array}$ & $\begin{array}{l}26.63 \\
25\end{array}$ & 7.5 \\
\hline 0.02 & $\begin{array}{l}0.033 \\
9 \\
\end{array}$ & $\begin{array}{l}0.007 \\
24 \\
\end{array}$ & $\begin{array}{l}17.10 \\
14 \\
\end{array}$ & $\begin{array}{l}25.69 \\
89 \\
\end{array}$ & 6.7 \\
\hline 0.04 & $\begin{array}{l}0.028 \\
1\end{array}$ & $\begin{array}{l}0.007 \\
56\end{array}$ & $\begin{array}{l}16.01 \\
98\end{array}$ & $\begin{array}{l}23.66 \\
56\end{array}$ & 5.7 \\
\hline
\end{tabular}

In this experiment computer simulations are carried out to evaluate the performance of Functional link neural network with exponential functional expansion for denoising of image corrupted with Salt and Pepper noise of mean zero and different variance. Here the 9 inputs of this network were the entries of $3 \mathrm{X} 3$ window of the noisy image and the target was the corresponding pixel value of the original image. 


\section{Experiment-2}

In this experiment its tried to cancel noise by using M-FLANN network. First the noisy Cameraman image is pass through the single layer neural network and the target be the original cameraman image. Image is corrupt with Gaussian noise of mean 0 and variance 0.01 . After passing through M-FLANN network the image obtain will be shown here. It is clear from result that image obtain is objectively better.

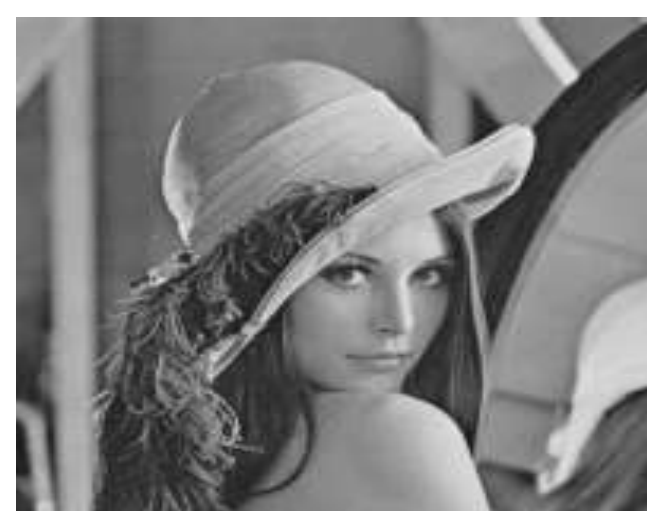

a) Original Image

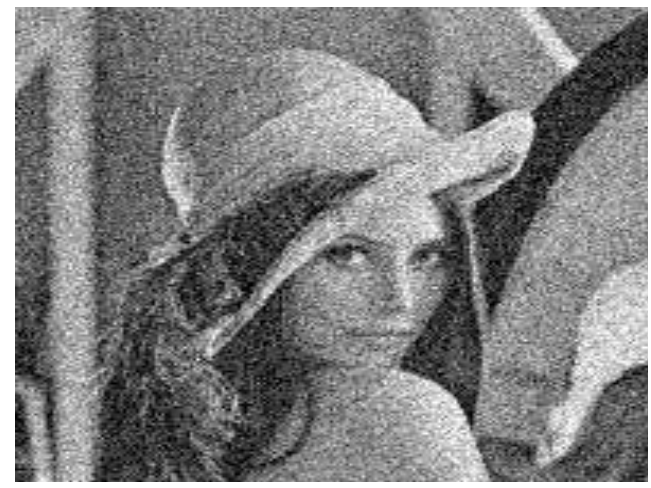

b) Noisy Image

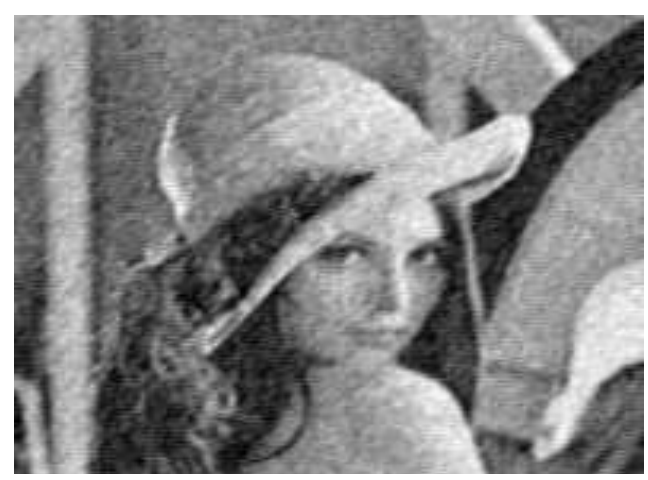

c) Image Pass Through M-FLANN

\section{Experiment3}

In this experiment computer simulations are carried out to evaluate the performance of Modified Functional link neural network with trigonometric functional expansion for denoising of image corrupted with Salt and Pepper Noise of 10 percent density. 


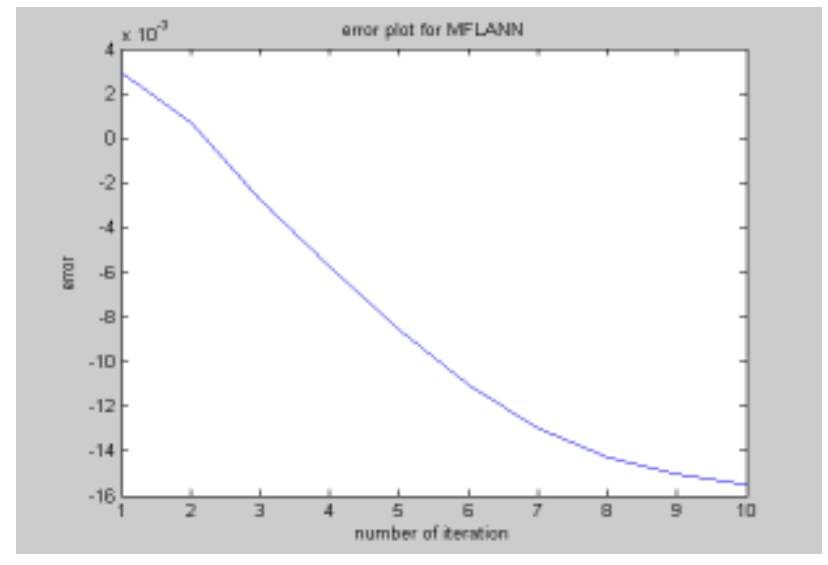

VI. Conclusion

Simulation results indicate that the performance of FLANN is better than MLP for Salt and Pepper noisesuppression from an image.It has better convergence speed than MLP. It has no hidden layer, is computationally cheap and has small number of interconnection weights and biases, which makes it suitable for on-line applications. Also, MFLANN makes use of linear terms, just as used in FLANNs and is able to approximate linear as well as nonlinear functions. From the results obtained in the work, it is concluded that the M-FLANN is computationally cheap and has small convergence times and denoise an digital image more efficiently than MLP.M-FLANN improves upon the approximation ability of FLANN. Therefore, it is expected that M-FLANN is likely to re-ignite the interest of research community in FLANNs.

\section{Future Work}

Better result will come out if we will improve the performance of the network by using efficient algorithms for color images processing in internet $\&$ wireless applications.

\section{References}

[1] Guez Allon, James L. Ellbert and Moshe Kam, "Neural networks architecture for control," IEEE Control System magazine, pp. 2225,1998 .

[2] Rivals Isabelle and Léon Personnaz, "Nonlinear internal model control using neural networks: Application to processes with delay and design issues," IEEE Trans. on Neural Networks, vol. 11, no.1, pp. 80-90, 2000.

[3] Yu Wen and Li Xiaoou, "Some new results on system identification with dynamic neural networks," IEEE Transactions on Neural Networks, vol.12, no. 2, pp. 412-417, 2001

[4] Naira Hovakimyan, Flavio Nardi, Anthony Calise, and Nakwan Kim, "Adaptive output feedback control of uncertain nonlinear systems using single-hiddenlayer neural networks," IEEE Trans. on Neural Networks, vol. 13, no. 6, pp. 1420-1431, 2002.

[5] Sunan N Huang., K. K. Tan and T. H. Lee, "Further results on adaptive control for a class of nonlinear systems using neural networks," IEEE Trans. on Neural Networks, vol. 14, no. 3, pp. 719-722, 2003.

[6] Sam Ge Shuzhi and Cong Wang, "Adaptive neural control of uncertain MIMO nonlinear systems," IEEE Trans. on Neural Networks, vol. 15, no. 3, pp. 674- 692, 2004

[7] S. E Lee and B. R. Holt, "Regression analysis of spectroscopic process data using a combined architecture of linear and nonlinear artificial neural networks," Neural Networks, vol. 4, pp. 549-554, 1992.

[8] Ying-Kai Zhao, Ning Cai, "DLF NEural network applied to process modelling and control," Proc. Pacific-Asian Conf. on Expert Systems PACES'95: Huangshan, PR China (1995) T.X Brown., "A high performance two-stage packet switch architecture,” IEEE Transactions on Communications, vol. 47, no. 8, pp. 1792-1795, 1999.

[9] Patra.J.C, Pal.R. N, Chatterji.B.N, Panda,G, "Identification of nonlinear dynamic systems using functional link artificial neural networks" IEEE Transactions, Systems, Man and Cybernetics, Part B,Vol 29, April-1999, pp 254 - 262.

[10] A.Namatame, and N.Ueda,"Pattern classification with Chebyshev neural networks," Ind.J.Neural Networks Vol 3,Mar. 1992, pp 2331

[11] Patra,J.C, Pal,R.N, "Functional link artificial neural network-based adaptive channel equalization of nonlinear channels with QAM signal" IEEE International Conference, Systems, Man and Cybernetics, 1995,Vol3, Oct.-1995, pp 2081-2086

[12] R Grino, G.Cembrano, and C.Torres, "Nonlinear system Identification using additive dynamic neural networks two on line approaches."IEEE Trans Circuits SystemIvol47, Feb 2000, pp 150-165.

[13] A.R.Foruzan, B.N.Araabi, "Iterative median filtering for restoration of images with impulsive noise." Electronics, Circuits and Systems, 2003. ICECS 2003.Dec 2003 ,PP 14-17 Dec.

[14] L. Corbalan, G.Osella, Massa.C.Russo, L.Lanzarini,. De Giusti 'Image Recovery Using a New Nonlinear daptive Filter Based on Neural Networks'’Journal of Computing and Information Technology - CIT 14, Apr.2006, pp 315-320. 